\title{
Dorsal Hippocampal Kindling Produces a Selective and Enduring Disruption of Hippocampally Mediated Behavior
}

\author{
Darren K. Hannesson, ${ }^{1}$ John Howland, ${ }^{2}$ Michael Pollock, ${ }^{3}$ Paul Mohapel, ${ }^{4}$ Amy E. Wallace,,${ }^{1}$ and \\ Michael E. Corcoran 1 \\ 1Departments of Psychology and Psychiatry, Neuropsychiatric Research Unit, University of Saskatchewan, Saskatoon, \\ Saskatchewan, Canada S7N 5E4, 22Department of Psychology, University of British Columbia, Vancouver, British \\ Columbia, Canada V6T 1Z4, 3Department of Psychology, Simon Fraser University, Vancouver, British Columbia, Canada \\ V5A 1S6, and "Section of Restorative Neurology, Wallenberg Neuroscience Center, University Hospital, S-221 85 Lund, \\ Sweden
}

Kindling produces enduring neural changes that are subsequently manifest in enhanced susceptibility to seizure-evoking stimuli and alterations in some types of behavior. The present study investigated the effects of dorsal hippocampal (dHPC) kindling on a variety of behaviors to clarify the nature of previously reported effects on spatial task performance. Rats were kindled twice daily with dHPC stimulation until three fully generalized seizures were evoked. Beginning $7 \mathrm{~d}$ later and on successive days, rats were tested in an elevated plus maze, a large circular open field, an open field object exploration task, and a delayed-match-to-place (DMTP) task in a water maze to assess anxiety-related and activity-related behavior (tasks 1 and 2), object recognition memory (task 3), and spatial cognition (task 4). Kindling disrupted performance on the DMTP task in a manner that was not delay dependent and produced a mild enhancement of activity-related behaviors in the open field task but not the elevated plus maze. All other aspects of testing were spared. These findings indicate that dHPC kindling produces enduring and selective effects on behavior that are consistent with a restricted disruption of hippocampally mediated functions. Possible bases for these effects are changes in local NMDA receptor function and/or changes in local inhibition, which might alter the optimal conditions for experiencedependent induction of intrahippocampal plasticity. This preparation may be useful for studying the mechanisms of mnemonic dysfunction associated with temporal lobe epilepsy and may offer unique insights into the mechanisms underlying normal hippocampal function.

Key words: activity; anxiety; CA1; DMTP; elevated plus maze; epilepsy; epileptogenesis; exploration; object recognition; open field; spatial; water maze
The repeated application of seizure-evoking stimulation (kindling) produces neural changes that are manifest in an enduring enhancement of susceptibility to seizure-evoking stimuli. A growing body of evidence also suggests that kindling may produce enduring alterations in some types of subsequent behavior (Depaulis et al., 1997; Adamec and Young, 2000; Hannesson and Corcoran, 2000; Kalynchuk, 2000; Leung et al., 2000). To identify their neural bases, it will be important to gain a better understanding of the nature of these behavioral effects.

Several studies have indicated that one type of behavior altered by kindling is that dependent on spatial cognition. Kindling disrupts performance in both the radial arm maze (Lopes Da Silva et al., 1986; Leung et al., 1990, 1996) and the Morris water maze (MWM) (Gilbert et al., 1996; Sutherland et al., 1997; Hannesson et al., 2001). Moreover, in the MWM, this effect is preferentially induced by kindling in the dorsal hippocampus (dHPC), by full rather than partial kindling, and is manifest in a disruption of learning and/or short-term memory- but not longterm memory-dependant performance (Hannesson and Corcoran, 2000; Hannesson et al., 2001). The interpretation of these

Received Nov. 3, 2000; revised March 2, 2001; accepted March 23, 2001.

This work was supported by a research grant awarded from Natural Sciences and Engineering Research Council of Canada (to M.E.C.).

Correspondence should be addressed to Michael E. Corcoran, Office of Research Services, University of Saskatchewan, 117 Science Place, Saskatoon, Saskatchewan, Canada S7N 5C8. E-mail: corcoran@admin.usask.ca.

Copyright (C) 2001 Society for Neuroscience 0270-6474/01/214443-08\$15.00/0 effects, however, is complicated because kindling also produces changes in non-mnemonic functions that might impact spatial task performance. For example, one of the best established behavioral effects of kindling is a change in anxiety-related behaviors (Adamec and Stark-Adamec, 1983; Adamec, 1990; Depaulis et al., 1997; Pinel et al., 1998; Kalynchuk, 2000), and dHPC kindling, specifically, has been shown to alter anxiety-related behaviors in the elevated plus maze (Kalynchuk et al., 1998) and defense-related behaviors on several different tests (Pinel et al., 1977). These findings highlight the importance of determining the contribution of non-mnemonic effects, and changes in anxietyrelated behaviors in particular, to the effects of dHPC kindling on spatial tasks.

Kindling has also been shown to affect performance on several aversive and nonaversive conditioning tasks (Hannesson and Corcoran, 2000). Although many of these effects have been observed after kindling in other sites, dHPC kindling specifically has been shown to alter acquisition performance on a Y-maze brightness discrimination task (Becker et al., 1997). Thus, it is important to determine whether the mnemonic effects of dHPC kindling are specific to tasks that require spatial cognition.

In the present study, we investigated the following issues related to the effects of dHPC kindling on spatial task performance: (1) the role of non-mnemonic effects, by assessing anxiety-related and activity-related behaviors in the elevated plus maze and an open field task, (2) the specificity of the mnemonic effects, by 
extending our analysis to an additional nonspatial task, an open field object recognition task, and (3) the relative impact on learning and short-term memory processes, by using a variable delay delayed-match-to-place procedure (DMTP) in the MWM.

\section{MATERIALS AND METHODS Subjects}

Twenty-six male Long-Evans hooded rats (Charles River Canada, St. Constant, Quebec, Canada) weighing 300-375 gm at the beginning of the study were used as subjects. Food and water were available ad libitum throughout the experiment. Rats were maintained in pairs in shoebox cages before surgery and were housed individually for the remainder of the experiment. All experimental procedures were performed during the light portion of the $12 \mathrm{hr}$ light/dark cycle. All rats were handled each day throughout the experiment except during the first $4 \mathrm{~d}$ after surgery. Subjects were randomly assigned to either the kindled group $(\mathrm{K})(n=13)$ or the control group $(\mathrm{C})(n=13)$. The 13 control rats were each yoked to one of the kindled rats.

\section{Surgery}

In preparation for surgery, animals were anesthetized with Somnotol (sodium pentobarbital; $60 \mathrm{mg} / \mathrm{kg}$ ) and given methyl scopolamine (1 $\mathrm{mg} / \mathrm{kg}$ ) to reduce respiratory congestion. Rats were placed in a stereotaxic apparatus, the skull was leveled, and a bipolar nichrome wire electrode $(127 \mu \mathrm{m}$ diameter) was implanted in either the right $(n=6)$ or left $(n=7)$ hippocampus using the following coordinates relative to bregma: anteroposterior, $-3.5 \mathrm{~mm}$; mediolateral, $2.6 \mathrm{~mm}$; dorsoventral, $-3.1 \mathrm{~mm}$. The electrode tips were separated by $0.4-0.5 \mathrm{~mm}$, with the lower tip used as the stimulating electrode. Five jeweler's screws were used to secure the electrode assembly to the skull, with one screw over the anterior neocortex serving as the reference electrode. The electrode assembly was affixed to the skull with dental acrylic, and a topical antibiotic-steroid, Topagen, was applied to the wound. Finally, a subcutaneous injection of Anafen $(0.5 \mathrm{cc} / \mathrm{kg})$ was given for postsurgical analgesia.

\section{Kindling}

Approximately $7 \mathrm{~d}$ after surgery, kindling was initiated. In the first kindling session, the stimulus intensity required to evoke an afterdischarge (AD) was determined. A Grass Instruments (West Warwick, RI) S8800 stimulator was used to deliver a $1 \mathrm{sec}$ train of balanced biphasic square wave $1 \mathrm{msec}$ pulses at 60 pulses per sec at an initial intensity of 1 $\mu \mathrm{A}$ (base-to-peak). If $\mathrm{AD}>4 \mathrm{sec}$ in duration was not evoked, intensity was increased along the following scale: $10,20,40,80,120,160$, and 250 $\mu \mathrm{A}$ every $2 \mathrm{~min}$ until $4 \mathrm{sec}$ or greater $\mathrm{AD}$ was elicited. The minimal intensity triggering $\mathrm{AD}$ was arbitrarily defined as $\mathrm{AD}$ threshold and was the intensity used for kindling during the remainder of the study. Subsequently, rats were kindled twice daily with stimulations separated by at least $4 \mathrm{hr}$ until a criterion of three stage 5 seizures was achieved. At this point, rats were considered fully kindled, and behavioral testing was started 7 or $8 \mathrm{~d}$ later. Each control rat was yoked to a specific kindled rat with which it received identical treatment, except that it was not stimulated.

\section{Behavioral assessment}

\section{Testing environment}

All testing took place in a rectangular windowless room with one door. The walls were painted an off-white color and were hung with numerous posters. Background noise was produced by an overhead ventilation fan. During all testing, the experimenter remained within the room at a computer station set up in one corner. For data acquisition, an overhead video camera coupled to a microcomputer by an image analyzer [Chromotrack (San Diego Instruments, San Diego, CA); EthoVision (Noldus Information Technology, Sterling, VA)] was used to track movement of rats in the various mazes. A remote switch was used to start and stop tracking, and a VCR was used to videotape most trials.

\section{Apparatus}

Elevated plus maze. The elevated plus maze was constructed from 19mm-thick plywood and corrugated plastic, which was used to line all areas of the maze that would be exposed to a rat during a trial. The maze consisted of two sets of perpendicular interlocking arms $110 \mathrm{~cm}$ in length and $10 \mathrm{~cm}$ in width. The interlocking central region bisected the maze into two pairs of arms, one with 45-cm-high walls, the closed arms, and one without walls, the open arms. The entire maze was elevated on legs that were $45 \mathrm{~cm}$ high.

Open field. The open field was made of a white industrial plastic and was painted white. It was circular in shape with $45-\mathrm{cm}$-high walls and a diameter of $150 \mathrm{~cm}$. Two identical objects, glass $500 \mathrm{ml}$ beakers with rings of black and white tape, were placed in the center of adjacent quadrants of the open field at a distance of $40 \mathrm{~cm}$ from the wall. For analysis purposes, the open field was divided into various regions: an outer ring (0-15 $\mathrm{cm}$ from the wall), a middle ring $(15-40 \mathrm{~cm}$ from the wall), an inner ring (40-75 $\mathrm{cm}$ from the wall), and two object zones $(30 \mathrm{~cm}$ in diameter centered on each object).

Object exploration task in the open field. The maze was identical to that used for the open field task except that novel objects, constructed from Lego (Billund, Denmark), were used. Each object was $\sim 8 \mathrm{~cm}$ high and 4 $\mathrm{cm}$ wide and was composed of pieces of three of the following different colors: red, blue, yellow, black, and white. For the first exploration trial, two identical objects were used. On the second exploration trial, an identical copy of the first object pair and a novel object were used.

Morris water maze. The Morris water maze was made of a white industrial plastic and was painted white. It was circular in shape with 45-cm-high walls and a diameter of $200 \mathrm{~cm}$. The maze was filled to a height of $26 \mathrm{~cm}$ with $22 \pm 1^{\circ} \mathrm{C}$ water rendered opaque with $1500-2000 \mathrm{ml}$ of skim milk powder. A clear Plexiglas platform $23 \mathrm{~cm}$ in height with $10 \times 12 \mathrm{~cm}$ upper face was used throughout the study. On visible platform trials, a black-sided attachment was added which caused the upper surface of the platform to protrude $3 \mathrm{~cm}$ above the surface of the water.

\section{Procedure}

Elevated plus maze. Activity (i.e., exploratory behaviors) and anxietyrelated behaviors were assessed in an elevated plus maze (Pellow et al., 1985; Rodgers and Dalvi, 1997). Rats were brought to the testing room and tested individually. Before each trial, the maze was cleaned thoroughly with a solution of $60 \%$ alcohol. The trial began with the rat being placed in the central region of the maze facing an open arm and continued for $5 \mathrm{~min}$, at which point the rat was promptly removed and returned to the housing colony. Data were obtained using tracking software and supplemented by experimenter observations, either live or from videotape. The following measures were taken. The time spent in each of the five regions of the maze (the two open arms, the two closed arms, and the central region) was recorded. For this purpose, the rat's position was determined according to the location of its center of gravity as indicated by the central point in the pixels representing the rat. Entries into each of the arms were recorded. For this purpose, the rat's entry to any of the four arms was counted each time all four paws crossed from the central region into an arm. Finally, the total distance traveled was recorded. Measures of activity included overall distance moved and total number of arm entries, with higher values on each measure indicating higher levels of activity. Measures of anxiety included dwell ratio, the ratio of dwell time in the open arms to the dwell time in all four arms, and entry ratio, the ratio of open arm to closed arm entries, with lower values indicating higher levels of anxiety.

Open field. Activity (i.e., exploratory behaviors) and anxiety-related behaviors were assessed in a modified open field task (Williams and Russel, 1972; Walsh and Cummins, 1976). This task also served as preparation for object exploration testing as described below. On each of 3 consecutive days, rats were brought to the testing room and tested individually. Before each trial, the maze and the objects were thoroughly cleaned with a solution of $60 \%$ alcohol. The trial began with the rat being placed in the maze at the opposite end from the objects facing the wall and continued for $5 \mathrm{~min}$, at which point the rat was promptly removed and returned to the housing colony. Data were obtained using the tracking software and supplemented by observations by the experimenter either live or from videotape. The following measures were taken. The time spent in each of the five regions of the maze [the three rings of the maze (outer, middle, and inner rings) and the two object areas] was recorded. For this purpose, the rat's position was determined according to the location of its center of gravity as indicated by the central point in the pixels representing the rat. Entries into the object regions were recorded. For this purpose, entries were counted each time both of the rat's front paws crossed the border of the region subsequent to being 
completely outside of the region. Rears were recorded each time the rat raised both front paws off the ground. Finally, the total distance traveled was recorded. Measures of activity included overall distance moved, time investigating the objects, and rears, with larger values on each measure indicating higher levels of activity. Measures of anxiety included the amount of time spent in the central region of the maze, with lower values indicating higher levels of anxiety.

Object exploration task in the open field. An object exploration task in the open field modified from Ennaceur et al. (1996) was used to assess object recognition memory. The $3 \mathrm{~d}$ of open field testing described above served as an opportunity for the rats to become habituated to the testing environment. After $1 \mathrm{~d}$ off, the critical trials were administered. Rats were brought to the testing room and tested individually. Before each trial, the maze and objects were thoroughly cleaned with a solution of $60 \%$ alcohol. The first exploration trial began with the rat being placed in the maze at the opposite end from the objects facing the wall and continued for $5 \mathrm{~min}$, at which point the rat was promptly removed and returned to the housing colony. The objects on this trial were identical. After $15 \mathrm{~min}$, the rat was returned to the testing room for the second object exploration trial. It was identical to the first trial, except that its duration was only $3 \mathrm{~min}$ and the objects were replaced by one identical copy and a novel object. On this trial, rats normally show a bias toward exploration of the novel object (Ennaceur et al., 1996). This, of course, requires that the rat recognizes the familiar object and therefore represents a test of object recognition memory. Data were obtained using the tracking software and were supplemented by observations by the experimenter either live or from videotape. The following measures were taken. The time spent in each of the five regions of the maze [the three rings of the maze (outer, middle, and inner rings) and the two object areas] was recorded. For this purpose, the rat's position was determined according to the location of its center of gravity as indicated by the central point in the pixels representing the rat. Entries into the object regions were recorded. For this purpose, entries were counted each time both of the rat's front paws crossed the border of the region subsequent to being completely outside of the region. Rears were recorded each time the rat raised both front paws off the ground. Finally, the total distance traveled was recorded. Measures of activity included overall distance moved, time investigating the objects, and rears. Measures of object recognition included dwell ratio, the time spent in the region of the novel versus the familiar object, and entry ratio, the entries into the region of the novel versus the familiar object, with larger values of each measure indicating better object recognition.

DMTP testing in the MWM. Spatial working memory was assessed using a DMTP procedure consisting of 10 consecutive days of testing. On each day, one trial group, consisting of a sample trial and three match trials, was administered with the platform location selected randomly without replacement from 1 of 10 possibilities. On the first day of testing, the visible platform was used, whereas, on subsequent days, the hidden platform was used. The delays between trials were constant within days and were as follows across the $10 \mathrm{~d}$ of testing: $0.25,0.25,0.25,0.25,0.25$, $1,4,0.25,1$, and $4 \mathrm{~min}$. The first $4 \mathrm{~d}$ of testing were considered the "acquisition phase" of the task on the basis of pilot data that had shown that rats' performance asymptoted by the fifth day of testing using the above protocol. The following $6 \mathrm{~d}$ were considered the "delay phase" of testing and were used to assess the impact of varying the intertrial interval on performance. For analysis purposes, data from each of the two trial groups at the same delay during this phase of testing were averaged.

Throughout testing, rats were brought to the testing room and tested in pairs (one kindled and one yoked control rat). For all trial groups, one "sample" trial and three "matching" trials were administered. On the sample trial, the rat was placed on the platform and allowed to remain there for a period of $20 \mathrm{sec}$, after which it was removed to a holding pen with a $250 \mathrm{~W}$ red heat lamp in the corner of the testing room. Previous work has shown that rats are able to acquire considerable information about a spatial location simply by viewing the environment from that location (Sutherland and Linggard, 1982). On the matching trials, the rat was returned to the pool and gently placed into the water facing the edge of the pool at a constant starting location (a spot on the "southernmost" wall of the pool proximal to the computer station) and allowed to swim until it found the platform or until $90 \mathrm{sec}$ elapsed, after which it was gently guided to the platform. The rat was allowed to remain there for 20 $\mathrm{sec}$ and was then returned to the holding pen. Two additional matching trials were administered as above, except that on the last trial the rat was removed from the platform immediately after the trial was completed.
Each matching trial was started at a delay of $0.25,1$, or 4 min after the removal of the rat from the platform on the preceding trial. On days 2-10, each trial group was preceded by a "free swim" in which no platform was present. The rat was placed in the pool at the usual starting position and permitted to swim until it passed over the exact location of the platform on the previous day of testing or until $30 \mathrm{sec}$ expired, at which point the experimenter removed the rat from the pool and returned it to the holding pen. Five minutes later, the DMTP trial group was started. In pilot work, this procedure was found to enhance performance by decreasing the likelihood that a rat would search for the platform in its location on the previous day of testing on subsequent training that day.

Data were obtained using the tracking software and were supplemented by observations by the experimenter either live or from videotape. The following measures were taken: latency to escape and directness of the swim path to the platform. A direct swim was recorded if the rat remained within a $25 \mathrm{~cm}$ alley between the start location and the platform.

\section{Kindling and behavioral testing schedule}

Starting $\sim 1$ week after surgery, rats were kindled twice daily until three stage 5 seizures were evoked. Seven days later, rats were tested in the elevated plus maze (day 7). The next $3 \mathrm{~d}$, rats were tested in the open field (days 8-10). After $1 \mathrm{~d}$ off, rats were tested in the object exploration task in the open field (day 12). After another day off, rats began $10 \mathrm{~d}$ of testing on the DMTP task (days 14-23). On day 1, training was completed using the visible platform and an intertrial delay of $0.25 \mathrm{~min}$. On days $2-4$, task acquisition was completed using the submerged platform and an intertrial delay of $0.25 \mathrm{~min}$. On days 5-10, memory testing was completed using the submerged platform and an intertrial delay of 0.25 , $1,4,0.25,1$, and 4 min on successive days.

\section{Histology}

After behavioral testing, animals were killed with an overdose of sodium pentobarbital or chloroform and perfused transcardially with $9 \%$ saline. Brains were fixed in formalin and then frozen before $60 \mu \mathrm{m}$ coronal sections were taken through the dHPC. Every section through the electrode track was mounted and stained with cresyl violet. The location of the electrode tips was documented by matching sections with one of four plates from Swanson (1992).

\section{Data analysis}

Data analysis was completed using the statistical software package SPSS for Windows (SPSS, Chicago, IL). Dependent measures from each of the behavioral tasks were subjected to analyses with repeated measures ANOVA and $t$ tests. Planned comparisons were made using $t$ tests. One-tailed tests were used when directional hypotheses guided analyses.

\section{RESULTS \\ Histology}

Electrodes in all kindled rats included in the study were located in the dHPC, and electrodes in all control rats included in the study were found in or near the dHPC. No gross histological changes were noted in the brains of either kindled or control rats other than gliosis around the electrode track.

\section{Kindling}

Kindling data were as follows. At a mean threshold of $35.4 \pm 3.3$ $\mu \mathrm{A}$, stimulation evoked an initial $\mathrm{AD}$ of $22.9 \pm 1.5 \mathrm{sec}$ in duration. In all cases, secondary AD was observed, although it was highly variable in its latency to onset and duration. A mean of $45.8 \pm 5.3$ stimulations was required to evoke the first stage 5 seizure, and $49.0 \pm 5.2$ stimulations were required to meet our kindling criterion of three stage 5 seizures. For the three stage 5 seizures, the mean AD duration, latency to clonus, and duration of clonus were $59.0 \pm 6.4,18.3 \pm 4.8$, and $24.8 \pm 1.4 \mathrm{sec}$, respectively. 
Figure 1. Measures of activity-exploration in the elevated plus maze. Kindled and control groups (both $n$ values $=13$ ) were tested $7 \mathrm{~d}$ after the completion of kindling. Data are presented as means \pm SEM. Total Entries, Total number of entries to all arms in the maze.
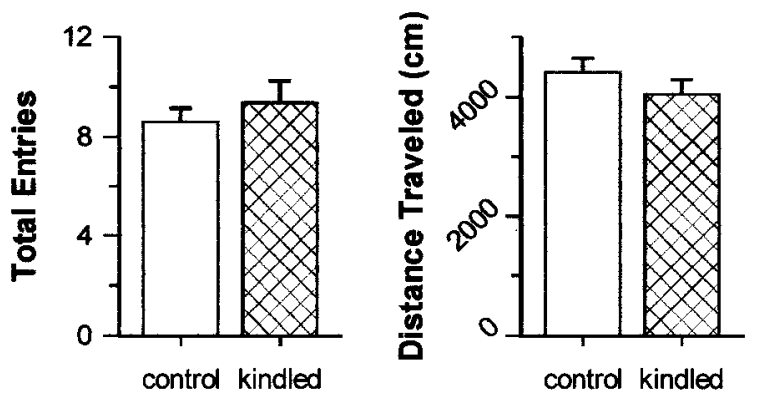

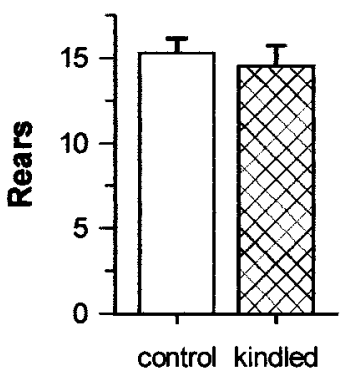

\section{Behavioral assessment}

\section{Elevated plus maze}

Full kindling of the dHPC did not significantly affect either activity- or anxiety-related behaviors in the elevated plus maze. Kindled and control rats exhibited comparable levels of activity as shown by similar distances traveled, total arm entries, and rears between groups (all $t_{(24)}$ values $<1.09 ; p$ values $>0.287$ ) (Fig. 1). Also, kindled and control rats exhibited comparable levels of anxiety, as shown by similar open arm dwell ratios and entry ratios (both $t_{(24)}$ values $=0.83$; $p$ values $\left.=0.412\right)($ Fig. 2$)$.

\section{Open field}

Similar to results in the elevated plus maze, full kindling of the dHPC did not significantly affect anxiety-related behaviors in the open field (Fig. 3). Kindled and control rats exhibited comparable levels of anxiety, as shown by similar dwell times in $\left(t_{(24)}=0.58\right.$; $p=0.569)$ and numbers of entries $\left(t_{(24)}=1.49 ; p=0.150\right)$ to the central ring of the open field.

However, in contrast to results in the elevated plus maze, some evidence suggests that full kindling of the dHPC did impact activity-related behaviors as assessed in the open field (Fig. 4). Kindled rats spent significantly greater amounts of time exploring the objects $\left(t_{(24)}=1.72 ; p=0.049\right.$; one-tailed $t$ test $)$ and reared more frequently $\left(_{(24)}=1.89 ; p=0.044\right.$; one-tailed $t$ test $)$ compared with control rats. However, kindled rats did not show significantly greater amounts of ambulation, as shown by comparable overall distance traveled by kindled and control rats $\left(t_{(24)}=\right.$ $0.72 ; p=0.476)$.

\section{Object exploration in the open field}

Full kindling of the dHPC did not significantly affect object recognition memory, as assessed in the object exploration in an open field task. On the first trial, with two copies of a novel object, kindled rats exhibited comparable amounts of activity-related (distance traveled, $t_{(24)}=0.72, p=0.476$; rears, $t_{(24)}=1.17, p=$


Figure 2. Measures of anxiety in the elevated plus maze. Testing took place $7 \mathrm{~d}$ after the completion of kindling. Data are presented as means \pm SEM. Entry Ratio, Number of open arm entries per number of closed arm entries; Dwell Ratio, time in open arms per trial duration.
$0.254)$ and anxiety-related (central ring dwell time, $t_{(24)}=0.59$; $p=0.563)$ behaviors relative to controls. Importantly, both groups also exhibited similar amounts of object exploration, as shown by comparable dwell times in $\left(t_{(24)}=0.02 ; p=0.983\right)$ and entries to $\left(t_{(24)}=1.66 ; p=0.111\right)$ the object regions. These data suggest that both groups had comparable opportunities to become familiar with the object used.

On the second trial, object recognition memory was assessed by replacing the objects from trial 1 with one identical copy of these objects and a novel object. Recognition memory should be reflected in a preference for exploring the novel object (Ennaceur et al., 1996). Kindled rats' performance was comparable with controls' in terms of both the ratio of novel to familiar object area entries $\left(t_{(24)}=0.52 ; p=0.611\right)$ and the ratio of novel to familiar object area dwell times $\left(t_{(24)}=1.80 ; p=0.084\right)$ (Fig. 5). Moreover, both groups approached the novel object more frequently than the familiar object, as shown by entries ratios that were biased toward the novel object, although only kindled rats' performance was significantly greater than chance on this measure (i.e., $>1$; kindled, $t_{(12)}=2.25, p=0.022$, one-tailed $t$ test; control, $t_{(12)}=1.385, p=0.111$, one-tailed $t$ test). Control rats also spent a significantly greater amount of time investigating the novel object, as suggested by a dwell times ratio that was significantly biased toward the novel object (i.e., $>1 ; t_{(12)}=3.29 ; p=0.003$; one-tailed $t$ test). However, this measure failed to reach statistical significance for kindled rats $\left(t_{(12)}=0.26 ; p=0.40\right.$; one-tailed $t$ test). Kindled rats' performance on all other measures, including distance traveled, rears, central ring dwell time, total object area dwell times, and total object area entries, did not significantly differ from that of control rats (all $t_{(24)}$ values $<1.01 ; p$ values $>$ 0.320). Collectively, these data suggest that kindling did not significantly disrupt object recognition memory.
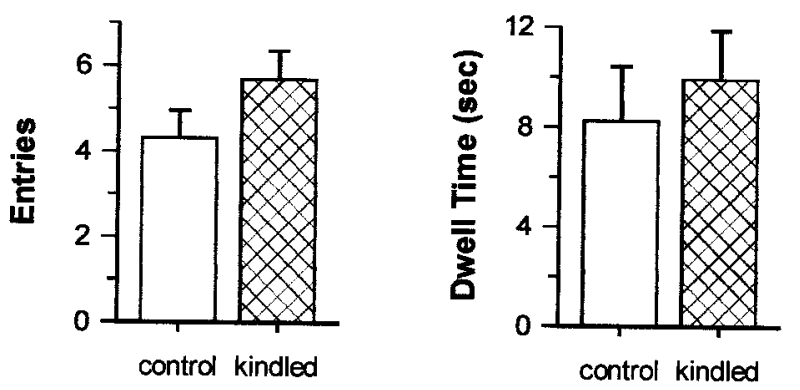

Figure 3. Measures of anxiety in the open field task. Data are from day 1 of open field testing, $8 \mathrm{~d}$ after the completion of kindling, and are presented as means \pm SEM. Entries, Number of entries to the central ring of the open field; Dwell Time, time spent in the central ring of the open field. 

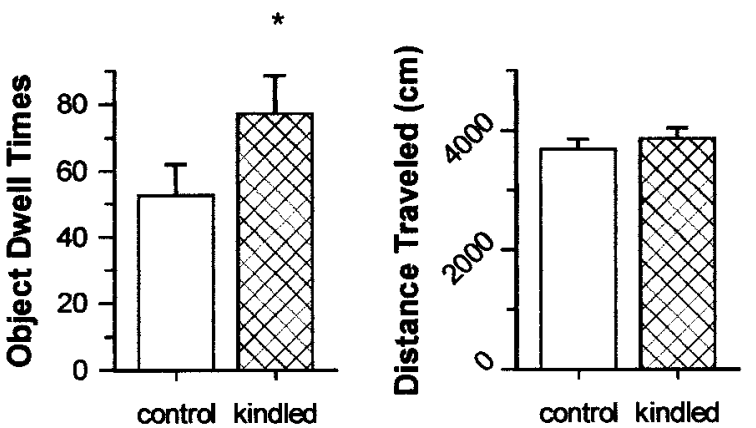

\section{Delayed-match-to-place in the MWM}

DHPC kindling did not affect performance on the DMTP task during the initial phase of testing (i.e., acquisition of the task). Kindled and control rats performed comparably across days of acquisition in terms of both escape latencies and direct swims (data not shown). This was evidenced by a lack of group effects (both $F_{(1,24)}$ values $<0.06$; both $p$ values $>0.592$ ), group $\times$ trial interactions (both $F_{(2,48)}$ values $<2.32$; both $p$ values $>0.109$ ), group $\times$ day interactions (both $F_{(2,48)}$ values $<0.03$; both $p$ values $>0.967$ ), and group $\times$ trial $\times$ day interactions (both $F_{(4,96)}$ values $<0.07$; both $p$ values $>0.659$ ) on either measure (data not shown). These results suggest that the groups did not differ in their initial abilities to perform the task.

However, dHPC kindling did significantly disrupt performance on the DMTP task during the delay phase of testing. Overall, kindled rats required longer latencies to escape and were less likely to take a direct swim path to the platform compared with controls, as shown by significant group effects in terms of both escape latencies $\left(F_{(1,24)}=5.697 ; p=0.025\right)$ (Fig. 6) and direct swims $\left(F_{(1,24)}=13.361 ; p=0.001\right)$ (Fig. 7$)$. Both groups did show significant improvements across trials, as indicated by a highly significant trial effect in terms of both escape latencies $\left(F_{(1.4,34.6)}\right.$ $=131.622 ; p<00.0005)$ and direct swims $\left(F_{(2,48)}=108.940 ; p<\right.$ $0.0005)$. However, there was some evidence that the groups differed in their performance across trials, as shown by a significant group $\times$ trials interaction in terms of direct swims $\left(F_{(2,48)}=\right.$ $3.269 ; p=0.047)$ but not escape latencies $\left(F_{(1.4,34.6)}=2.083 ; p=\right.$ $0.151)$. Analysis of simple main effects of group within trials in terms of direct swims indicated that kindled rats performed more poorly than controls on trials 2 and 3 (both $F_{(1,24)}$ values $>5.01$; both $p$ values $<0.035)$ but not trial $1\left(F_{(1,24)}=0.50 ; p=0.489\right)$.
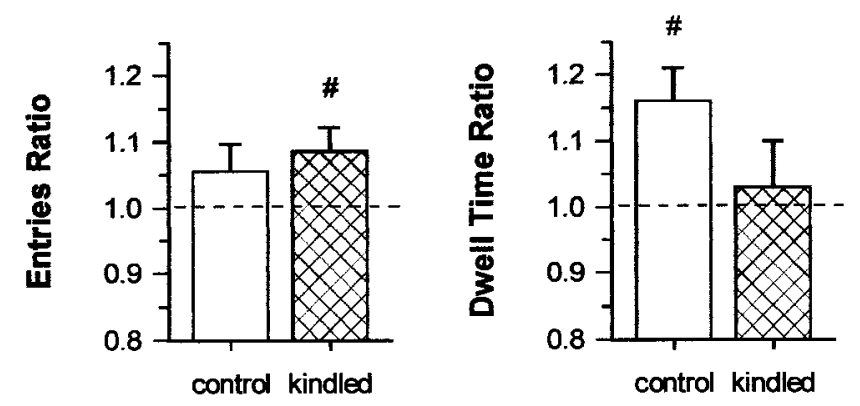

Figure 5. Performance on measures of object recognition memory in the object exploration open field task, $12 \mathrm{~d}$ after the completion of kindling. Data are means \pm SEM. Dashed line represents chance levels of performance. Entries, Ratio of number of bouts of exploration of the novel object per number of bouts of exploration of the familiar object. Dwell Time, Ratio of time spent exploring the novel object per time spent exploring the familiar object. $\# p<0.05$ relative to chance performance.
For comparison, the same analyses were performed on the escape latency data, and a similar pattern of results was found (trials 2 and 3 , both $F_{(1,24)}$ values $>11.39$, both $p$ values $<0.003$; trial 1 , $\left.F_{(1,24)}=0.01, p=0.975\right)$. Although both groups tended to perform better at shorter delays, the delay effect was not significant in terms of either escape latencies $\left(F_{(2,48)}=2.191 ; p=0.123\right)$ or direct swims $\left(F_{(2,48)}=2.576 ; p=0.087\right)$, nor were the delay $\times$ group (both $F_{(2,48)}$ values $<0.538$; both $p$ values $>0.587$ ), delay $\times$ trial, or delay $\times$ group $\times$ trial interactions significant in terms of either measure $\left(\right.$ all $F_{(4,96)}$ values $<1.357$; all $p$ values $\left.>0.255\right)$. Planned comparisons between groups suggested that kindled and control rats performed comparably on trial 1 at each delay (direct swims, all $t_{(24)}$ values $<0.68$, all $p$ values $>0.10$, one-tailed $t$ test; latency, all $t_{(24)}$ values $<0.61$, all $p$ values $>0.10$, one-tailed $t$ test) and on trial 3 at the $60 \mathrm{sec}$ delay, at least in terms of escape latency $\left(t_{(24)}=0.78 ; p<0.10\right.$, one-tailed $t$ test). In contrast, kindled rats performed significantly worse than controls on trial 2 at all delays (direct swims, all $t_{(24)}$ values $>2.29$, all $p$ values $<$ 0.01 , one-tailed $t$ test; latency, all $t_{(24)}$ values $>1.77$, all $p$ values $<$ 0.05 , one-tailed $t$ test) and on trial 3 at the 15 and $240 \mathrm{sec}$ delays (direct swims, all $t_{(24)}$ values $>1.63$, all $p$ values $<0.05$, one-tailed $t$ test; latency, all $t_{(24)}$ values $>1.85$, all $p$ values $<0.05$, one-tailed $t$ test). The difference between the groups on trial 3 at the $60 \mathrm{sec}$ delay also approached significance, at least in terms of direct swims $\left(t_{(24)}=1.06 ; p<0.10\right.$; one-tailed $t$ test $)$.

\section{DISCUSSION}

In the present study, the behavioral effects of full kindling of the dHPC were assessed in an elevated plus, an open field task, an object exploration task in an open field, and a DMTP task in the MWM. Kindling disrupted DMTP performance in a delayindependent manner and increased some activity-related behaviors in the open field but did not affect any other aspect of performance.

This is the first study to show that full kindling of the dHPC does not affect anxiety-related behaviors in either the elevated plus maze or an open field task. Kindled rats explored the open arms of the elevated plus maze and the central region of the open field as freely as control rats. Both of these are well validated measures of anxiety or fearfulness in rodents, and the consistent finding of no effect across the tasks argues strongly that full dorsal hippocampal kindling did not affect anxiety-related behaviors or emotionality in general. This finding contrasts with previous research suggesting that dHPC kindling may increase anxietyemotionality in rats (Pinel et al., 1977; Kalynchuk et al., 1998). The major difference between these and the present findings is that extended kindling rather than full kindling was used. Thus, the extent of kindling may be an important determinant of the anxiogenic effects of kindling. This hypothesis is consistent with 
Figure 6. Latency to escape to the hidden platform on matching trials $1(A), 2(B)$, and $3(C)$ during delay phase of testing on the DMTP task in the MWM, 18-23 d after kindling. Data are averaged across $2 \mathrm{~d}$ of testing at each delay and are presented as means \pm SEM. ${ }^{*} p<0.05$ relative to control performance.

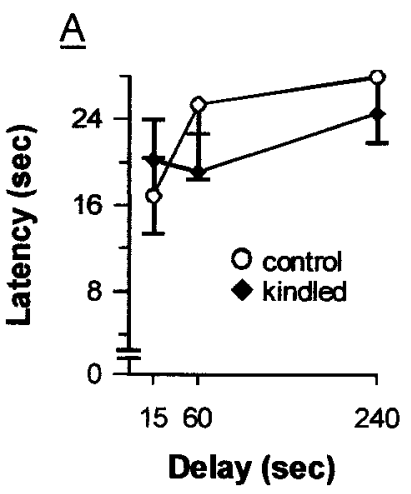

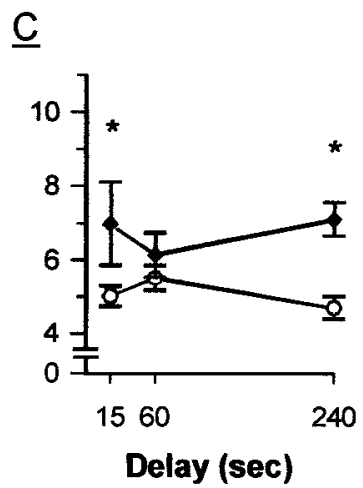

observations that extent of amygdala kindling correlates positively with the magnitude of its effects on anxiety-emotionality (Kalynchuk et al., 1997).

The present study is also the first to show that full dHPC kindling affects activity-exploration. Kindled rats reared more frequently and spent more time exploring objects in the open field relative to control rats. However, they did not show increased activity-exploration on other measures in the open field or on any measure in the elevated plus maze. The discrepancy of the effect between tasks might be explained by the observation that richer environments typically elicit greater amounts of activityexploration and hence are more likely to be sensitive to small changes in such behaviors (Hall, 1956). In our case, the open field had a much larger surface area and contained a greater variety of stimuli (e.g., the objects) than the elevated plus maze and thus may have been more sensitive to a slight effect of kindling on activity-exploration. Increased activity-exploration is generally consistent with the pattern of effects of hippocampal lesions on open field behavior (O'Keefe and Nadel, 1978) and thus could indicate a disruption of hippocampal function. This interpretation is consistent with the hypothesis that hippocampal dysfunction also underlies impaired spatial task performance after kindling (Lopes Da Silva et al., 1986; Leung and Shen, 1991; Leung et al., 1990, 1994, 1996; Sutherland et al., 1997; Hannesson et al., 2001) and presents a possible explanation for the effects of dHPC kindling on brightness discrimination task performance (Becker et al., 1997). Because the task is sensitive to the effects of hippocampal lesions (Munoz and Grossman, 1981; McLamb et al., 1988), the effect of kindling may also be a result of a direct alteration of hippocampal function.

The present study provides additional evidence that nonspatial forms of cognition are spared by full dHPC kindling. Kindled rats' performance was comparable with controls' on both measures of object recognition in the open field object exploration task. This is consistent with our previous finding that full dHPC kindling spares object associative memory (Hannesson et al., 2001) and suggests that object-related memory in general is unaffected by dHPC kindling. Thus, the type of information processing required by a task (e.g., spatial versus object related) is a critical determinant of the susceptibility of a task to disruption by dHPC kindling. Other task dimensions, such as the workingreference memory demands, may be of less significance because kindling disrupts both spatial reference (Lopes Da Silva et al., 1986; Gilbert et al., 1996; Hannesson et al., 2001) and spatial working memory tasks (Leung et al., 1990, 1994, 1996; Leung and Shen, 1991; Sutherland et al., 1997).

The main finding of the present study is that full kindling of the dHPC disrupted DMTP performance in the MWM. Kindled rats were significantly slower in escaping to the hidden platform and used less direct routes on matching trials 2 and 3 at all three delays tested $(0.25,1$, and $4 \mathrm{~min})$. This result suggests that full dHPC kindling produces an anterograde impairment of spatial working memory function. However, an impairment of other mnemonic functions or non-mnemonic functions that may have affected nonspatial components of task performance might account for the deficit. Several considerations argue against this possibility. First, both kindled and control rats rapidly improved on the task and did not differ during the acquisition phase of testing. Second, both kindled and control rats performed comparably on the first matching trial at all delays. Third, we have shown previously that dHPC-kindled rats perform normally on a visible platform control task in the MWM (Gilbert et al., 1996), even when tested before any other water maze experience (Hannesson et al., 2001). Finally, kindled rats performed normally on most other aspects of performance in the other tasks assessed in the present study, suggesting that their basic behavioral repertoires were unaffected by kindling. In particular, the failure of kindling to alter anxiety-related behaviors in either the elevated
Figure 7. Direct swims to the hidden platform on matching trials $1(A), 2(B)$, and $3(C)$ during delay phase of testing on the DMTP task in the MWM, $18-23 \mathrm{~d}$ after kindling. Data are averaged across $2 \mathrm{~d}$ of testing at each delay and are presented as means \pm SEM. A direct swim was scored if the rat remained within a $25 \mathrm{~cm}$ alley from the start position to the platform. ${ }^{*} p<0.05$ relative to control performance.
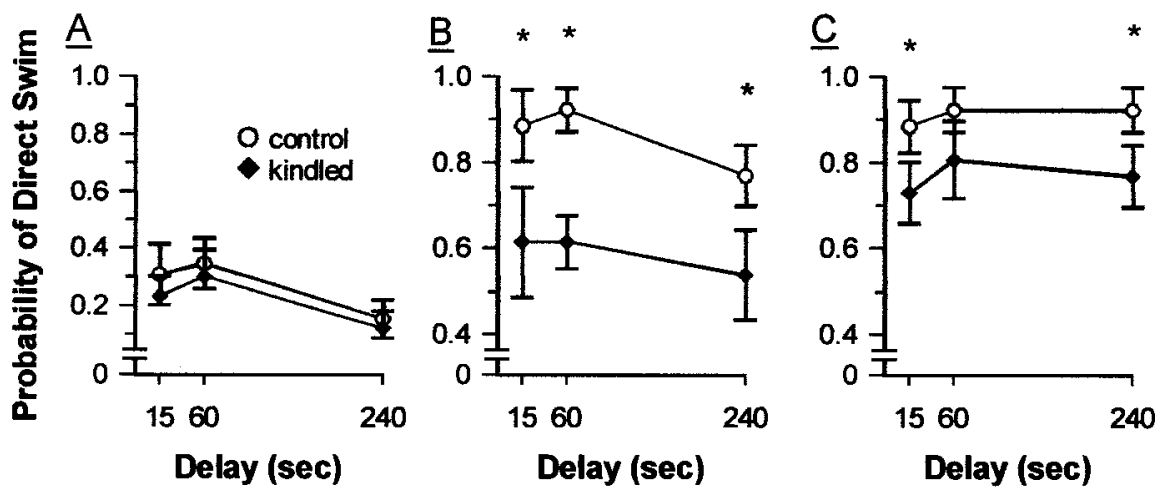
plus maze or open field eliminates anxiety-related factors as a basis for altered DMTP performance.

The absence of a relationship between the intertrial delay and the deficit suggests that the impairment is more likely one of spatial learning than short-term memory. If spatial learning were intact and short-term memory affected, one would predict that performance should deteriorate more quickly relative to controls across longer delays because these would place progressively greater demands on short-term memory function. The absence of a delay-dependent deficit is thus not consistent with the presence of a short-term memory deficit and suggests, in the absence of a non-mnemonic basis for the deficit, that learning was disrupted. However, given that the shortest delay used was $15 \mathrm{sec}$, it is still possible that an impairment in short-term memory underlies the observed effect, but it would have to be one that reaches asymptotic levels within that time period.

An additional point that can be derived from the present DMTP findings is that the kindling-induced impairment of spatial cognition is quite long lasting. Testing on the DMTP task began $14 \mathrm{~d}$ after the completion of kindling, and the delay phase of testing took place over a period of $6 \mathrm{~d}$ from 18-23 d after the completion of kindling. This is the longest interval between dHPC kindling and testing at which an anterograde impairment of spatial cognition has been detected. Some evidence suggests that the impairment produced by dHPC kindling may dissipate by 28 d after kindling (Sutherland et al., 1997). However, in this study, partial rather than full kindling was used, and there was intervening training before $28 \mathrm{~d}$ testing, which confounds recovery with relearning. Thus, it remains unclear whether the anterograde impairment produced by full dHPC kindling dissipates over any time period.

Based on the present findings, a characterization of the effects of kindling on spatial cognition can be formulated, which should help identify possible underlying mechanisms for this effect. Kindling produces a specific profile of behavioral impairments that is most consistent with a direct disruption of hippocampal function. Furthermore, this disruption appears to be most likely manifest in impaired spatial learning processes and is long lasting for a period of at least $14 \mathrm{~d}$ after the last kindling stimulation. Thus, the mediating mechanisms for kindling-induced effects on spatial cognition should (1) directly and selectively induce hippocampal dysfunction, (2) selectively compromise functions involved in learning, not short- or long-term memory, and (3) be long lasting. We hypothesize, then, that they are localized to hippocampal circuitry, affect the induction but not maintenance or expression of plasticity, and are an enduring consequence of kindling, particularly in the dHPC.

Two mechanisms that meet these criteria are a disruption of NMDA receptor function and a disruption of inhibitory mechanisms. DHPC kindling has been shown to produce changes in NMDA receptor currents (Mody, 1999) and changes in both inhibition and $\mathrm{GABA}_{\mathrm{A}}$ receptor characteristics (de Jonge and Racine, 1987; Titulaer et al., 1995; Mody, 1999) that last for 1 month or longer after kindling. Because these changes might be expected to alter the optimal physiological conditions for induction of intrahippocampal forms of plasticity, such as long-term potentiation, they could disrupt the strength, pattern, or effectiveness of plastic changes related to learning. According to this hypothesis, then, kindling may represent an enduring form of metaplasticity (Abraham and Tate, 1997), which results in a shift away from the optimal "settings" for intrahippocampal plasticity and hence hippocampal-dependent learning.
In summary, the present study has shown that full kindling of the dHPC produces a profile of behavioral effects that is consistent with a mild disruption of hippocampal function. These results further highlight the selective effects of dHPC kindling on spatial cognition and characterize this model as a useful means to study epilepsy-related mnemonic dysfunction. They also suggest that kindling may produce metaplastic effects that result in an enduring dysfunction in the mechanisms that mediate hippocampal-dependent functions.

\section{REFERENCES}

Abraham WC, Tate WP (1997) Metaplasticity: a new vista across the field of synaptic plasticity. Prog Neurobiol 52:303-323.

Adamec R (1990) Amygdala kindling and anxiety in the rat. NeuroReport 1:255-258.

Adamec R, Young B (2000) Neuroplasticity in specific limbic system circuits may mediate specific kindling induced changes in animal affect: implications for understanding anxiety associated with epilepsy. Neurosci Biobehav Rev 24:705-723.

Adamec RE, Stark-Adamec C (1983) Limbic kindling and animal behavior: implications for human psychopathology associated with complex partial seizures. Biol Psychiatry 18:269-293.

Becker A, Letzel K, Letzel U, Grecksch G (1997) Kindling of the dorsal and the ventral hippocampus: effects on learning performance in rats. Physiol Behav 62:1265-1271.

de Jonge M, Racine RJ (1987) The development and decay of kindlinginduced increases in paired-pulse depression in the dentate gyrus. Brain Res 412:318-328.

Depaulis A, Helfer V, Deransart C, Marescaux C (1997) Anxiogeniclike consequences in animal models of complex partial seizures. Neurosci Biobehav Rev 21:767-774.

Ennaceur A, Neave N, Aggleton JP (1996) Neurotoxic lesions of the perirhinal cortex do not mimic the behavioural effects of fornix transection in the rat. Behav Brain Res 80:9-25.

Gilbert TH, McNamara RK, Corcoran ME (1996) Kindling of hippocampal field CA1 impairs spatial learning and retention in the Morris water maze. Behav Brain Res 82:57-66.

Hall JF (1956) The relationship between external stimulation, food deprivation and activity. J Comp Physiol Psychol 49:339-341.

Hannesson DK, Corcoran ME (2000) The mnemonic effects of kindling. Neurosci Biobehav Rev 24:725-751.

Hannesson DK, Mohapel P, Corcoran ME (2001) Dorsal hippocampal kindling selectively impairs spatial learning/short-term memory in the MWM. Hippocampus, in press.

Kalynchuk LE (2000) Long-term amygdala kindling in rats as a model for the study of interictal emotionality in temporal lobe epilepsy. Neurosci Biobehav Rev 24:691-704.

Kalynchuk LE, Pinel JPJ, Treit D, Kippin TE (1997) Changes in emotional behavior produced by long-term amygdala kindling in rats. Biol Psychiatry 41:438-451.

Kalynchuk LE, Pinel JPJ, Treit D (1998) Long-term kindling and interictal emotionality in rats: effects of stimulation site. Brain Res 779:149-157.

Leung LS, Shen B (1991) Hippocampal CA1 evoked response and radial 8-arm maze performance after hippocampal kindling. Brain Res 555:353-357.

Leung LS, Boon KA, Kaibara T, Innis NK (1990) Radial maze performance following hippocampal kindling. Behav Brain Res 40:119-129.

Leung LS, Zhao D, Shen B (1994) Long-lasting effects of partial hippocampal kindling on hippocampal physiology and function. Hippocampus 4:696-704.

Leung LS, Brzozowski D, Shen B (1996) Partial hippocampal kindling affects retention but not acquisition and place but not cue tasks on the radial arm maze. Behav Neurosci 110:1017-1024.

Leung LS, Ma J, McLachlan RS (2000) Behaviors induced or disrupted by complex partial seizures. Neurosci Biobehav Rev 24:763-775.

Lopes Da Silva FH, Gorter JA, Wadman WJ (1986) Kindling of the hippocampus induces spatial memory deficits in the rat. Neurosci Lett 63:115-120.

McLamb RL, Mundy WR, Tilson HA (1988) Intradentate colchicine impairs acquisition of a two-way active avoidance response in a Y-maze. Neurosci Lett 94:338-342.

Mody I (1999) Synaptic plasticity in kindling. In: Jasper's basic mechanisms of the epilepsies, Ed 3, Advances in neurology, Vol 79 (DelgadoEscueta AV, Wilson WA, Olsen RW, and Porter RJ, eds), pp 631-643. Philadelphia: Lippincott-Williams \& Wilkins.

Munoz C, Grossman SP (1981) Spatial discrimination, reversal and active or passive avoidance learning in rats with KA-induced neuronal depletions in dorsal hippocampus. Brain Res Bull 6:399-406.

O'Keefe J, Nadel L (1978) The hippocampus as a cognitive map. Oxford: Clarendon. 
Pellow S, Chopin P, File SE, Briley M (1985) Validation of open-closed entries in an elevated plus-maze: a novel test of anxiety in the rat. J Neurosci Methods 14:149-167.

Pinel J, Treit D, Rovner LI (1977) Temporal lobe aggression in rats. Science 197:1088-1089.

Pinel JPJ, Kalynchuk LE, Treit D (1998) Long-term amygdala kindling and defensive behavior in rats. In: Kindling 5, Vol 48 (Corcoran ME, Moshe SL, eds), pp 349-358. New York: Plenum.

Rodgers RJ, Dalvi A (1997) Anxiety, defense and the elevated plusmaze. Neurosci Biobehav Rev 21:801-810.

Sutherland RJ, Linggard R (1982) Being there: a novel demonstration of latent spatial learning in the rat. Behav Neural Biol 36:103-107.

Sutherland RJ, Leung LS, Weisend MP, Schlife J, McDonald RJ
(1997) An evaluation of the effect of partial hippocampal kindling on place navigation by rats in the Morris water task. Psychobiology 25:126-132.

Swanson LW (1992) Brain maps: structure of the rat brain. New York: Elsevier.

Titulaer MN, Ghijsen WE, Kamphuis W, De Rijk TC, Lopes da Silva FH (1995) Opposite changes in GABAA receptor function in the CA1-3 area and fascia dentata of kindled rat hippocampus. J Neurochem 64:2615-2621.

Walsh RN, Cummins RA (1976) The open-field test: a critical review. Psychol Bull 83:482-504.

Williams DI, Russel PA (1972) Open-field behavior in rats: effects of handling, sex and repeated testing. Br J Psychol 63:593-596. 